\title{
Mixed honeycomb pushing refinement
}

\author{
Huining Meng ${ }^{\mathrm{a}}$, Hartmut Prautzsch ${ }^{\mathrm{b}, *}$ \\ ${ }^{a}$ Hangzhou, Dianzi University, China \\ ${ }^{b}$ Karlsruher Institute of Technology (KIT), 76131 Karlsruhe, Germany
}

\begin{abstract}
We generalize the honeycomb scheme, dualize it and combine both the primal and the dual scheme into self-dual subdivision schemes for convex polyhedra which generate $\mathcal{C}^{1}$ surfaces without line segments different from the honeycomb scheme, which generates $\mathcal{C}^{1}$ surfaces having line and even planar segments.
\end{abstract}

\section{Introduction}

Corner cutting refers to a special class of curve or surface generating (geometric) subdivision schemes, where in the curve case, a polygon $\mathcal{P}$ is replaced iteratively by an interpolating polygon, i. e., by a polygon whose vertices lie on (the edges of) $\mathcal{P}$ meaning that we cut and replace parts of $\mathcal{P}$ by new edges. Chaikin's [7] or the more general de Rham scheme [19] and de Casteljau's algorithm or its more general projective version by Haase $[12,3]$ are such corner cutting schemes.

It is well-known that polygons generated by corner cutting converge to continuous curves [4] and for local corner cutting, where every cut raises the number of vertices, we even have a simple characterization of all schemes that generate $\mathcal{C}^{1}$ curves $[5,16]$. However, similar results have not yet been published for surfaces generated by corner cutting except partly for [6] and the fact that some authors use the term corner cutting in a loose sense for any iterative replacement (of the vertices) of control meshes by (the vertices of) finer meshes with not necessarily planar faces.

Generalizing $[4,5,10,16]$ and following [6], we use the term corner cutting only in its strict sense, where it means for (solid) polyhedra that we chop of parts by

\footnotetext{
*Corresponding author

Email address: prautzsch@kit.edu (Hartmut Prautzsch)
} 
planar cuts, i. e., corner cutting means to iteratively intersect more and more half spaces to generate a convex body.

Interestingly, no corner cutting scheme for polyhedra has been published so far except for the algorithms in $[20,13]$ and one that only if dualized could be interpreted as a corner cutting scheme. It is the honeycomb scheme by Dyn, Levin and Liu [9] which is an interpolating convexity preserving refinement scheme for convex polyhedra generating $\mathcal{C}^{1}$ surfaces in the limit. Thus there are tangent planes for all vertices and these could be used to define local cuts of the tangent polyhedra with specified contact points [6]. The iterative insertion of contact elements, i. e., tangent planes with contact points, is what is called a fill-in-scheme in [16] and fill-in-schemes are dual (in the sense of projective geometry) to fill-in-schemes.

Honeycomb surfaces have a dense set of planar segments, which makes them interesting mathematical objects but less desirable in, e. g., class A surface design and for stability reasons as planar metal sheets tend to bend back and forth.

These facts: (1) that honeycomb surfaces have interesting special shape characteristics or artefacts, (2) that the primal honeycomb algorithm has been the only interpolatory convexity preserving $\mathcal{C}^{1}$ scheme for closed surfaces, and (3) that the dual honeycomb algorithm has been the only candidate for a surface corner cutting scheme inspired us to investigate the honeycomb algorithm (1) to develop tools that could help to (better) understand corner cutting for surfaces, (2) to modify the honeycomb algorithm so as to get rid of the planar and line segments, and (3) to come up with first genuine corner cutting schemes for polyhedra.

In fact, we found a way to modify the honeycomb algorithm into a self-dual scheme generating convex $\mathcal{C}^{1}$ surfaces without line (or planar) segments and present the results in this paper. Furthermore, our investigations helped us to finally understand corner cutting for surfaces and sparked first corner cutting algorithms [17].

Before we present our modification of the honeycomb scheme, we recall the honeycomb scheme, compare it to other honeycomb schemes and show why it generates surfaces with planar segments.

\subsection{The standard honeycomb scheme}

Given a closed, strictly convex polyhedron $\mathcal{P}_{0}$, the standard honeycomb scheme generates a sequence of polyhedra $\mathcal{P}_{i}$ in the following way:

For each face $F$ of $\mathcal{P}_{i}$, we build one face of $\mathcal{P}_{i+1}$, called an $f$-face, and for each edge of $\mathcal{P}_{i}$, we build one face of $\mathcal{P}_{i+1}$ called an $e$-face. These two kinds of faces are shown in Figure 1 and are formed as follows. 


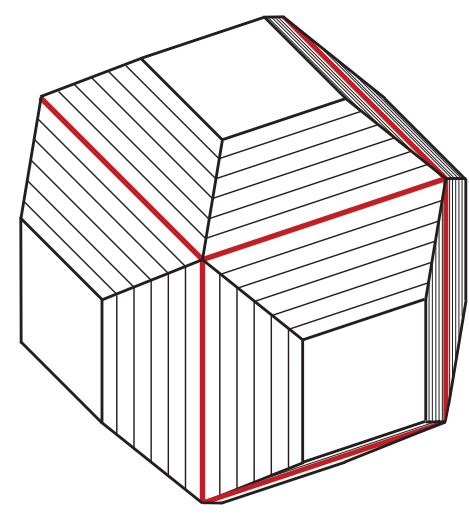

Figure 1: A step of the honeycomb scheme applied to a cube (shown in red) gives a polyhedron with 6 quadrilateral $f$-faces (white) and 12 hexagonal $e$-faces (hatched).

To each edge of $\mathcal{P}_{i}$, we assign the exterior bisecting plane of the two adjacent faces. Each face $F$ of $\mathcal{P}_{i}$ and all the bisecting planes corresponding to its edges form a closed polyhedron $\triangle_{F}$ outside $\mathcal{P}_{i}$. The $f$-face $F^{\prime}$ of $\mathcal{P}_{i+1}$ associated to $F$ is the intersection of the polyhedron $\triangle_{F}$ with the midplane between $F$ and the nearest vertex $\mathbf{v}_{F}$ of $\triangle_{F}$, that is not on $F$. Note that the midplane is parallel to $F$.

The polyhedron $\triangle_{F}$ trimmed by $F^{\prime}$ has 4 -sided faces besides the faces $F$ and $F^{\prime}$. Each bisecting plane has two such 4 -sided faces. Together they form a hexagonal face, which is the $e$-face of $\mathcal{P}_{i+1}$ assigned to the edge common to both 4 -sided faces.

We remark that associated $f$-faces have the same valence while all $e$-faces are hexagonal. Thus all polyhedra $\mathcal{P}_{i}$ have equally many non-valence- 6 faces and roughly four times as many hexagonal faces as their predecessors $\mathcal{P}_{i-1}$. This is a typical property shared by other subdivision algorithms as, e. g., the half box spline subdivision algorithm [18] and its extension [2]. Topologically, they generate the same edge graphs. We note that hexagonal meshes can also be subdivided in a way that is dual topologically to the $\sqrt{3}$-refinement of triangular meshes [14]. Three such algorithms are the algorithms in [1, 8, 22] which are all stationary subdivision algorithms and no genuine corner cutting algorithms in the strict sense. A genuine corner cutting algorithm with a $\sqrt{3}$-refinement topology is presented in $[17,20]$.

\subsection{The (general) honeycomb scheme $\mathrm{H}$}

As observed in [9], we can generalize the standard honeycomb scheme in the following way: Instead of bisecting planes, we take any planes dividing the exterior angles such that the ratios are in some interval $\left(\varrho, \varrho^{-1}\right)$ where $\varrho=$ 
$\epsilon:(1-\epsilon)>0$ with $\epsilon \in(0,1 / 2]$ is the honeycomb tolerance and instead of offsetting faces $F$ halfway towards the next vertex $\mathbf{v}_{F}$, we offset them to any position between $F$ and $\mathbf{v}_{F}$. In this paper, this generalization is called just the honeycomb scheme. The honeycomb scheme and the operation that transforms a polyhedron $\mathcal{P}_{i}$ into the next polyhedron $\mathcal{P}_{i+1}$ are denoted by the same letter $\mathrm{H}$.

We call a sequence $\left(\mathcal{P}_{i}\right)_{i \in \mathbb{N}}$ generated by $\mathrm{H}$ a honeycomb or $\mathrm{H}$ sequence. Figure 2 shows honeycomb surfaces generated from a cuboid and an icosahedron with $\varrho=1 / 2$ and different face offsetting percentages $\tau$ defining to which percentage faces are moved towards their extreme positions. For all surfaces shown in this paper, we used fixed ratios and offsetting distances. However without convincing results, we also tried to choose the ratios and offsetting distances for each edge and face independently of each other so as to obtain more uniform edge lengths and face areas. In Section 3, we show that the honeycomb surfaces are continuously differentiable for any random choice of all ratios, the honeycomb tolerance $\rho$ and all offsetting distances.

In general, the honeycomb scheme generates surfaces with planar segments as we see from the following example.

\subsection{An Example}

First we consider a polyhedron with a regular hexagonal face having equal angles with its 6 neighbors. Hence the six bisectors (of the hexagon and its neighbors) intersect at a common point above the center of the hexagon. Thus for the standard honeycomb scheme, the new $f$-face parallel to the hexagon is a regular hexagon of half the size and if we continue subdividing, we obtain a sequence of parallel $f$-faces which are scaled versions of the initial hexagon, where the scaling factor is always $1 / 2$ as can be seen in Figure 3(a).

Next we stretch every second edge of the hexagon by, e.g., doubling their length which we call $l$ while we keep the same angles. Then we obtain the situation shown in Figure 3(b). The short edges of the parallel $f$-faces are as long as before while all long edges are longer by the same additive constant $l$.

In the $i$-th subdivision step, the bisectors of a short edge and its two long neighbors intersect in a point. There are three such points, which we call $\mathbf{a}_{i}$, $\mathbf{b}_{i}$, $\mathbf{c}_{i}$, such that all $\mathbf{a}_{i}$ (or $\mathbf{b}_{i}$ or $\mathbf{c}_{i}$ ) have the same orthogonal projection $\mathbf{a}$ (or $\mathbf{b}$ or $\mathbf{c}$, respectively). Hence the limiting surface contains the triangle with edge length 1 spanned by $\lim _{i \rightarrow \infty} \mathbf{a}_{i}, \lim _{i \rightarrow \infty} \mathbf{b}_{i}$ and $\lim _{i \rightarrow \infty} \mathbf{c}_{i}$.

\subsection{Outline of this paper}

Our goal is to modify the honeycomb scheme $\mathrm{H}$ so as to obtain $\mathcal{C}^{1}$ surfaces without line segments in the limit. As we explain in Section 2, the dual honeycomb 


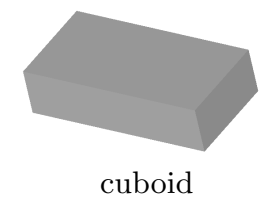

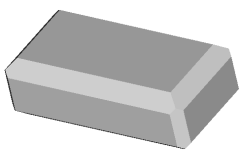

$\mathrm{H}_{20}^{1}$

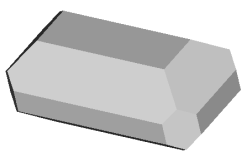

$\mathrm{H}_{50}^{1}$

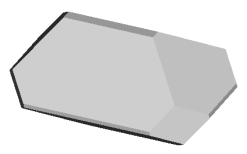

$\mathrm{H}_{90}^{1}$

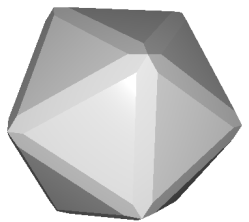

$\mathrm{H}_{20}^{1}$

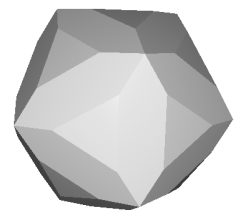

$\mathrm{H}_{50}^{1}$

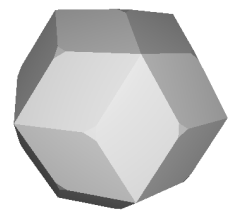

$\mathrm{H}_{90}^{1}$

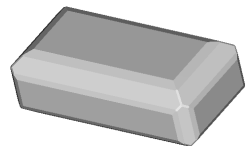

$\mathrm{H}_{20}^{2}$

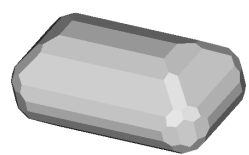

$\mathrm{H}_{50}^{2}$

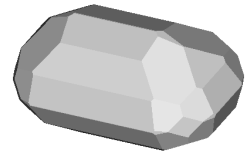

$\mathrm{H}_{90}^{2}$

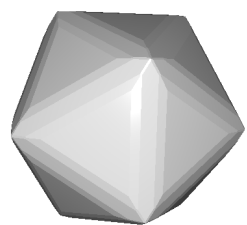

$\mathrm{H}_{20}^{2}$

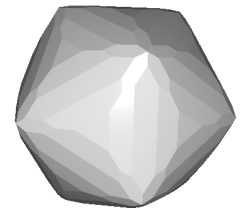

$\mathrm{H}_{50}^{2}$

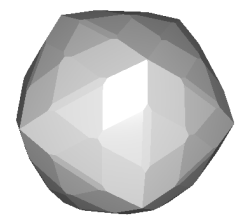

$\mathrm{H}_{90}^{2}$

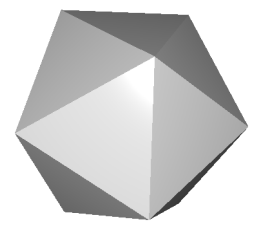

icosahedron

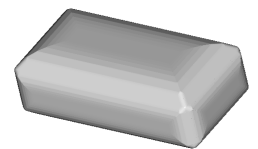

$\mathrm{H}_{20}^{7}$

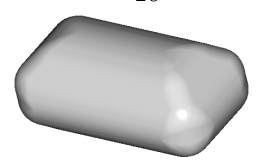

$\mathrm{H}_{50}^{7}$

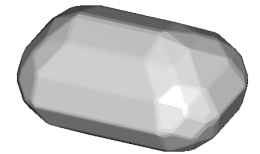

$\mathrm{H}_{90}^{7}$

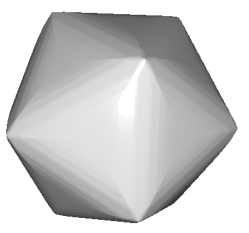

$\mathrm{H}_{20}^{7}$

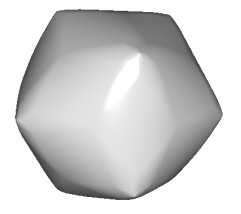

$\mathrm{H}_{50}^{7}$

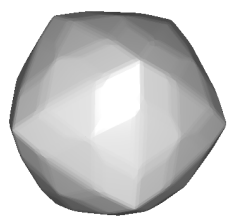

$\mathrm{H}_{90}^{7}$

Figure 2: A cuboid and an icosahedron refined by 1, 2 and 7 iterations of the honeycomb scheme with offsetting percentages 20,50 and 90 from top to bottom. 


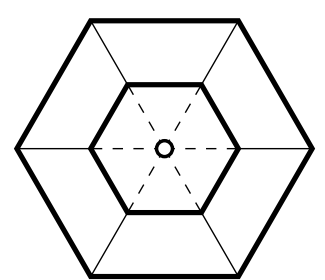

$(a)$

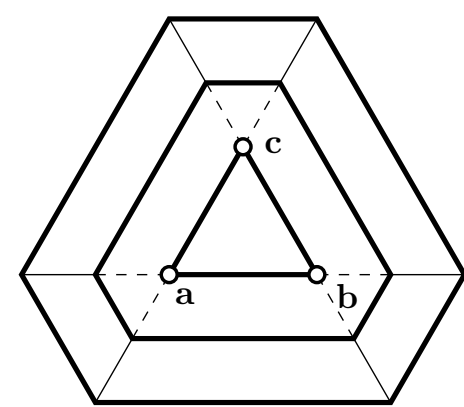

(b)

Figure 3: The honeycomb scheme applied to two hexagons with equal exterior angles at their edges.

scheme called the pushing scheme $\mathrm{P}$ generates limiting surfaces without line segments. However, these surfaces can contain corners. Therefore our idea is to combine the primal and the dual honeycomb scheme by applying alternately certain numbers of primal and dual honeycomb steps. In Section 5, we will show that such a mixed or MHP scheme generates $\mathcal{C}^{1}$ surfaces without line segments.

As a guide for our paper, Figure 4 shows how the theorems and other facts depend on each other where an asterisk indicates dualization.

\section{Basics}

We call a point $\mathbf{p}$ in $\mathbb{R}^{3} \backslash\{\mathbf{o}\}$ and the plane $\mathbf{p}^{*}$ given by the equation $\mathbf{p}^{t} \mathbf{x}=1$ dual to each other. (In the projective extension of $\mathbb{R}^{3}$, we can also dualize the origin $\mathbf{o}$. It is dual to the ideal plane.) Dualizing the points of a closed bounded convex surface $S$ enclosing the origin, we obtain the supporting planes of the dual surface $S^{*}$ which is also closed, bounded, convex and encloses the origin.

In particular, a point $\mathbf{p}$ of $S$ and a supporting plane through $\mathbf{p}$ are dual to a supporting plane with a contact point on $S^{*}$. Thus, a planar segment or a face of $S$ is dual to a corner of $S^{*}$ with a 2-parameter family of supporting planes. Further, a line segment of $S$ is dual to a point of $S^{*}$ with a 1-parameter family of supporting planes. It is called a pinch point, see Figure 5 . Note that $S$ can be a polyhedron and that the faces of a polyhedron are dual to the vertices of the dual polyhedron.

2.1 Assumption (Polyhedra contain the origin). In this paper, a polyhedron is considered to be a non-degenerate finite convex body in $\mathbb{R}^{3}$ containing the origin as an interior point. Further we assume that the boundary of a polyhedron consists of planar faces that are pair-wise non-coplanar. Hence, its vertices are 


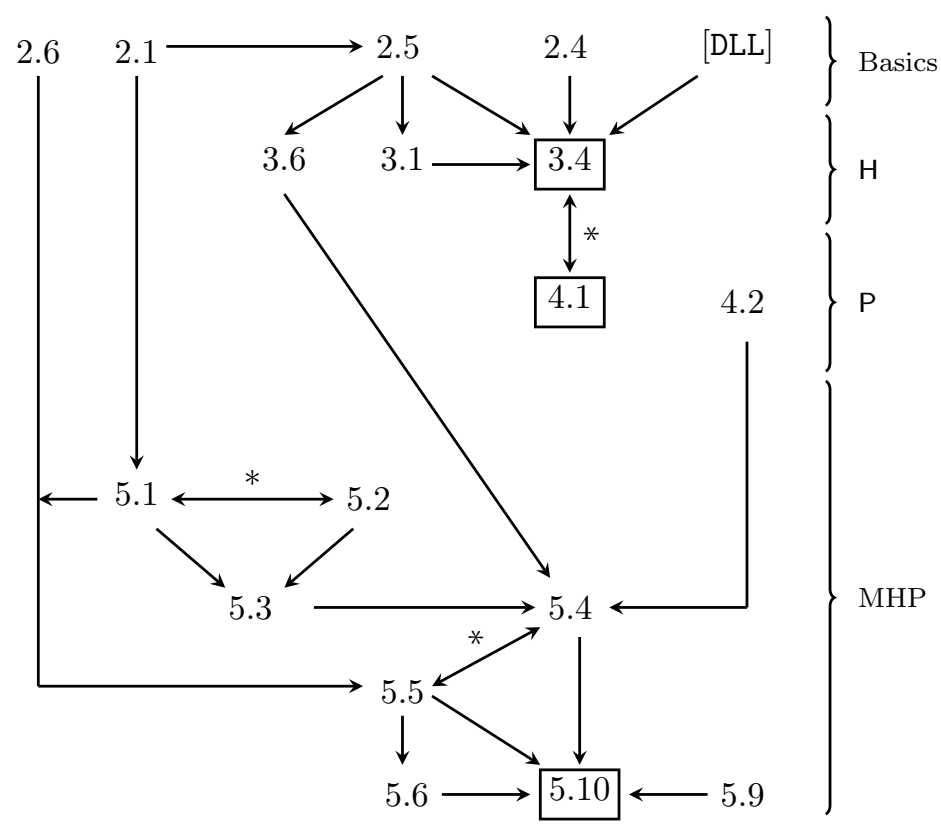

2.1 Assuming non-degenerate polyhedra.

2.4 Honeycomb sequences are bounded.

$2.5 \mathrm{H}$ keeps vertex angles below some $\alpha<180^{\circ}$.

2.6 Edges are boundedly longer than their dual angles.

$3.1 \mathrm{H}$ flattens the maximum vertex angle geometrically.

$3.4 \mathrm{H}$ surfaces are $C^{1}$.

$3.6 \mathrm{H}$ flattens the maximum face $p$-angle geometrically.

4.1 P-surfaces have no line segment.

$4.2 \mathrm{P}$ does not increase face angles.

5.1 MHP surfaces enclose open balls.

$5.2 \mathrm{MHP}$ sequences are bounded.

5.3 MHP keeps vertex angles below some $\alpha<180^{\circ}$.

5.4 MHP flattens the maximum face angle geometrically.

5.5 MHP shrinks the maximum edge length geometrically.

5.6 MHP sequences converge.

5.9 MHP patch normals are controlled by face 6 -rings.

5.10 MHP surfaces are $C^{1}$ and have no line segments.

Figure 4: The proof stucture where asterisks denote dualization. 


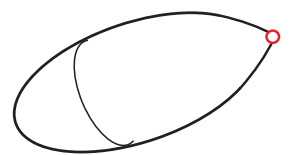

corner

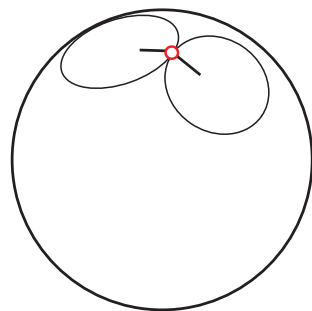

pinch point

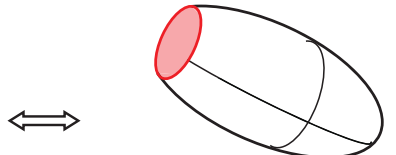

planar segment

Figure 5: Dualizing surfaces with a corner or pinch point.

the intersections of three or more faces. They are the extreme points of the convex body.

2.2 Definition (The Gaussian image of a polyhedron). Let $\mathcal{P}$ be a polyhedron. The normals of its supporting planes containing an edge of $\mathcal{P}$ determine a spherical edge, i. e., a segment of a great circle of the unit sphere $S^{2}$ and all these spherical edges form a mesh, which we call the Gaussian image of $\mathcal{P}$ and denote it by $\mathcal{P}^{\circ}$. Note that the exterior angle of any edge in $\mathcal{P}$ equals the length of its associated or dual edge in $\mathcal{P}^{\circ}$.

2.3 Definition (Vertex, edge and face angles). For every vertex $\mathbf{v}$ of a polyhedron $\mathcal{P}$, we define the vertex angle $\alpha_{\mathbf{v}}$ as the maximum angle of two supporting planes going through $\mathbf{v}$, for every edge $E$ of $\mathcal{P}$, we call the exterior angle of the two adjacent faces the edge angle $\alpha_{E}$ and for every face $F$ of $\mathcal{P}$, we define the face angle $\alpha_{F}$ as the maximum angle of two supporting planes of $\mathcal{P}$ going through two vertices of $F$. Note that $\alpha_{\mathbf{v}}$ is the spherical diameter of the face in $\mathcal{P}^{\circ}$ corresponding to $\mathbf{v}$ and that $\alpha_{F}$ is the spherical diameter of the vertex 1-ring of $\mathcal{P}^{\circ}$ around the normal vector $\mathbf{n}$ of $F$, which consists of all faces in $\mathcal{P}^{\circ}$ with vertex $\mathbf{n}$. We denote the spherical diameter of any subset $U$ of the unit sphere $\mathcal{S}^{2}$ by

$$
s \text {-diam } U:=\max \{\text { spherical distance }(\mathbf{p}, \mathbf{q}) \mid \mathbf{p}, \mathbf{q} \in U\} .
$$

2.4 Lemma. Any honeycomb sequence $\left(\mathcal{P}_{i}\right)_{i \in \mathbb{N}}$ is bounded.

Proof in a nutshell. The angles at parallel edges shrink geometrically as $i \rightarrow \infty$ and bound the honeycomb construction.

Proof. Consider any face $F$ of $\mathcal{P}_{1}$ and its associated $f$-faces $F_{i}$ on the $\mathcal{P}_{i}$. The edge angles of these faces $F_{i}$ shrink at least by the factor $(1-\epsilon)=1 /(1+\varrho)$ 
where $\varrho$ is the honeycomb tolerance as we increase $i$ by one. Thus for sufficiently large $i$ all edge angles of all $F_{i}$, where $F$ is any face of $\mathcal{P}_{1}$, are less than $90^{\circ}$. Hence, the polyhedra $\triangle_{F_{i}}$ introduced in Section 1.1 are bounded and contain all $F_{j}, j \geq i$. Thus, the polyhedra $Q_{j}$ that are parallel to $\mathcal{P}_{1}$ and whose faces contain the $F_{j}$ form a bounded sequence converging to a bounded polyhedron $Q_{\infty}$ which contains all $\mathcal{P}_{i}$.

2.5 Remark (A bound for the vertex angles of an $\mathrm{H}$ sequence). Let $\left(\mathcal{P}_{i}\right)_{i \in \mathbb{N}}$ be a honeycomb sequence and let $\alpha_{i}$ be the maximum vertex angle of $\mathcal{P}_{i}$. Because of Assumption 2.1 and since any face of $\mathcal{P}_{i+1}^{\circ}$ is contained in a face of $\mathcal{P}_{i}$, we see that $\pi>\alpha_{1} \geq \alpha_{2} \geq \alpha_{3} \geq \cdots$.

2.6 Remark (Bounding dual lengths). Under dualization, angles between adjacent faces become lengths of edges, where the length depends continuously on the faces and changes if we move the faces. All sequences of polyhedra considered in this paper are bounded with faces bounded away from the origin. Hence the quotient of angles between adjacent faces and their dual length lies in some bounded interval $\left[\sigma, \sigma^{-1}\right] \subset(0, \infty)$.

\section{Honeycomb surfaces are smooth}

In [9] it is shown that the angles around every vertex shrink to zero under the honeycomb scheme. Here, we show that they shrink uniformly and geometrically.

3.1 Theorem (H flattens the maximum vertex angle). Let $\left(\mathcal{P}_{i}\right)$ be a honeycomb sequence and let $\mu_{i}$ be the maximum vertex angle of $\mathcal{P}_{i}$. Then there are constants $c>0$ and $\nu \in(0,1)$ such that

$$
\mu_{i} \leq c \nu^{i} .
$$

Proof in a nutshell. The Gaussian images $\mathcal{P}_{i}^{\circ}$ are generated by a dual honeycomb scheme on $S^{2}$ where any face $G$ of $\mathcal{P}_{i+k}^{\circ}$ lies in some face $F$ of $\mathcal{P}_{i}^{\circ}$ for $k>0$. Using a central projection $\pi$, we project $F$ and $G$ into a suitable plane and observe for $k \geqslant$ valence $F$ that $\operatorname{diam} \pi G \leq(1-\delta)^{k} \operatorname{diam} \pi F$ where $\delta \in(0,1)$ is the number for the projected dual scheme corresponding to the honeycomb constant $\epsilon$. Taking into account the stretching of ratios by the central projection, we arrive at the theorem.

Proof. The spherical polyhedra $\mathcal{P}_{i}^{\circ}$ are generated by a spherical dual honeycomb scheme where all edges lie on great circles of $\mathcal{S}^{2}$. Under this scheme every edge is split in a ratio $\in\left[\varrho, \varrho^{-1}\right]$ and any face determined by the normals of $\mathcal{P}_{i}$ at some vertex $\mathbf{p}$ of valence $v$ is subdivided into $v$ triangles and a face of the same valence determined by the normals of $\mathcal{P}_{i+1}$ at $\mathbf{p}$ as illustrated in Figure 6 . 


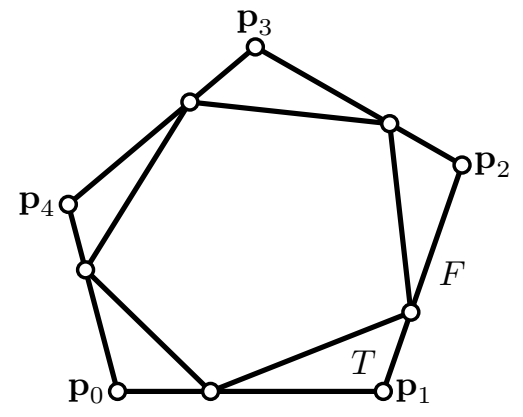

Figure 6: The spherical dual honeycomb scheme applied to a face $F$.

We project every face $F$ of $\mathcal{P}_{i}^{\circ}$ by a central projection $\pi$ from the origin into the tangent plane of $\mathcal{S}^{2}$ at some point $\mathbf{c}$ of $F$. Under any such projection $\pi$, the ratios defining the new faces with respect to the old ones change. Because of Remark 2.5, a continuity argument shows that they stay in some interval $\left[\bar{\varrho}, \bar{\varrho}^{-1}\right] \subset(0, \infty)$.

For the projected faces, we will show that the face diameters shrink geometrically. This implies that they shrink similarly on the sphere since the projection stretches lengths within a face $F$ by a bounded factor. So let $\overline{\mathbf{p}}_{0}, \ldots, \overline{\mathbf{p}}_{k}$ be the vertices of a planar polygon $\bar{F}$ of valence $v=k+1$. We subdivide it $k=v-1$ times by the planar version of the dual honeycomb scheme with subdivision ratios in the interval $\left[\bar{\varrho}, \bar{\varrho}^{-1}\right]$. Any triangle $\bar{T}$ generated within $\bar{F}$ lies in a triangle formed by two adjacent edges of $\bar{F}$ scaled by $1-\bar{\epsilon}=\bar{\varrho} /(1+\bar{\varrho})$. For example in Figure 6 , triangle $T$ lies in triangle $\mathbf{p}_{0} \mathbf{p}_{1} \mathbf{p}_{2}$.

Hence

$$
\operatorname{diam} \bar{T} \leq(1-\bar{\epsilon}) \operatorname{diam} \bar{F}
$$

For the non-triangular faces within $\bar{F}$, we consider the face $\bar{G}$ obtained after $k$ subdivision steps and denote its vertices by $\overline{\mathbf{q}}_{0}, \ldots, \overline{\mathbf{q}}_{k}$. The $\overline{\mathbf{q}}_{i}$ are convex combinations of the $\overline{\mathbf{p}}_{j}$ and depend on all of them, i. e.,

$$
\overline{\mathbf{q}}_{i}=\sum_{j=0}^{k} \overline{\mathbf{p}}_{j} \omega_{j}=\overline{\mathbf{p}}_{0}+\sum_{j=1}^{k}\left(\overline{\mathbf{p}}_{j}-\mathbf{p}_{0}\right) \omega_{j}
$$

where, e. g., $\omega_{0}=1-\sum_{j=1}^{k} \omega_{j} \geq \bar{\epsilon}^{k}$. Hence $\bar{G}$ lies in a scaled version of $\bar{F}$ and $\operatorname{diam} \bar{G} \leq\left(1-\bar{\epsilon}^{k}\right)$ diam $\bar{F}$, which concludes the proof.

Theorem 3.1 and Lemma 2.4 are slightly stronger than Proposition 4 and Proposition 5 in [9]. Therefore and since all other proofs in [9] are also valid in the general case, we obtain the following theorem: 
3.4 Theorem (Honeycomb surfaces are smooth). The limiting surfaces generated by the general honeycomb scheme are $C^{1}$ surfaces.

In Section 5, we need the stronger version of Theorem 3.1 below which we prepare by the following definition of $p$-angles:

Let $\left(\mathcal{P}_{i}^{\circ}\right)_{i \in \mathbb{N}}$ be the Gaussian image of a honeycomb sequence and let $\alpha$ be its largest vertex angle. From Remark 2.5, we recall that $\alpha<\pi$. Further we note that any vertex 1-ring, say $R$, of $\mathcal{P}_{i}^{\circ}, i \in \mathbb{N}$, lies in some vertex 1-ring, say $R_{j}$, of $\mathcal{P}_{j}^{\circ}$ for $j=1, \cdots, i$.

We call the intersection of $\mathcal{S}^{2}$ with a halfplane a $\boldsymbol{c a p}$ and define its diameter to be the length of any great circle segment splitting it symmetrically. For example, semispheres are caps with diameter $\pi$. An extreme example not relevant for this paper is $\mathcal{S}^{2}$ itself, which is a cap with diameter $2 \pi$.

If $R$ lies in a closed spherical cap of diameter $\alpha$, we define $Q$ to be the largest $R_{j}$ still contained in such a cap, project $R$ into the plane that goes through the cap's boundary relative to $\mathcal{S}^{2}$, which is a circle on $\mathcal{S}^{2}$, and denote the projection by $\bar{R}$. With $F$ being the face of $\mathcal{P}_{i}$ dual to the central vertex of $R$, we define

$$
p \text {-angle } F:=p \text {-diam } R:=\operatorname{diam} \bar{R} \text {. }
$$

If $R$ does not lie in a cap of diameter $\alpha$, we define its $p$-diameter as its spherical diameter, i. e.,

$$
p \text {-angle } F:=p \text {-diam } R:=s \text {-diam } R=\text { face angle } \alpha_{F} \text {. }
$$

Similarly we define the $p$-diameters for the vertex 1-rings if $\left(\mathcal{P}_{i}\right)_{i \in \mathbb{N}}$ is not a honeycomb sequence but still has the property that any vertex 1-ring of the $\mathcal{P}_{i}^{\circ}$ is contained in some 1-ring of $\mathcal{P}_{1}^{\circ}$.

3.5 Remark. Under the projection above, any distance $d$ in a spherical cap with diameter $\alpha$ changes by a factor in some interval $\left[\sigma, \sigma^{-1}\right] \subset(0, \infty)$. Hence

$$
\sigma \cdot \alpha_{F}=\sigma \cdot s \text {-diam } R \leq p \text {-diam } R \leq \sigma^{-1} \cdot s \text {-diam } R=\sigma^{-1} \cdot \alpha_{F},
$$

where we note that a diameter is not projected into a diameter in general.

3.6 Theorem ( $\mathrm{H}$ shrinks p-angles). Let $\left(\mathcal{P}_{i}\right)$ be a honeycomb sequence and let $\mu_{i}$ be the largest $p$-angle of $\mathcal{P}_{i}$. Then there is a constant $\gamma \in(0,1)$ such that

$$
\mu_{i+k} \leq \gamma \mu_{i}
$$

for all $i \in \mathbb{N}$, where $2 k-1$ or $2 k-2$ is the maximum vertex valence of $\mathcal{P}_{1}$.

Note that the maximum vertex valence is the same for all $\mathcal{P}_{i}$ and that it equals the maximum face valence of all $\mathcal{P}_{i}^{\circ}$. 
Proof in a nutshell. The vertex 1-rings $R$ of the Gaussian images $\mathcal{P}_{i}^{\circ}$ are contained in the interior of a vertex 1-ring $Q$ of $\mathcal{P}_{i-2}^{\circ}$ or $\mathcal{P}_{i-k}^{\circ}$ as shown in Figures 7 and 8 , and since $s$-diam $R<s$-diam $Q$, the quotient $(s$-diam $R) /(s$-diam $Q)$ stays below some constant less than 1 over the compact set of all 1-rings with an $s$-diameter $\geq \alpha$. For diameters $\in(0, \alpha]$, we project the 1-rings as above into a plane in which the quotient of diameters does not depend on the scale and where it suffices to consider the 1-rings with, e.g., diameter $\alpha / 2$. These form a compact set which implies again Equation 3.7.

Proof. We note that a honeycomb operation $\mathrm{H}$ does not increase the $p$-diameters of the vertex 1-rings in the Gaussian images $\mathcal{P}_{i}^{\circ}$, which are generated by a spherical dual honeycomb scheme where every edge is split in some ratio in $\left[\varrho, \varrho^{-1}\right]$ with $\varrho$ being the honeycomb tolerance.

To show that these diameters shrink as claimed in Equation 3.7, we distinguish two cases:

Case 1. First, we consider the vertex 1-rings $R_{2}$ of $\mathcal{P}_{i+2}^{\circ}$ that consist only of triangles and are contained in a vertex 1-ring $R$ of $\mathcal{P}_{i}^{\circ}$ : There are four types of these "triangle vertex 1-rings" as illustrated in Figure 7 where we marked a 1-ring of every type.

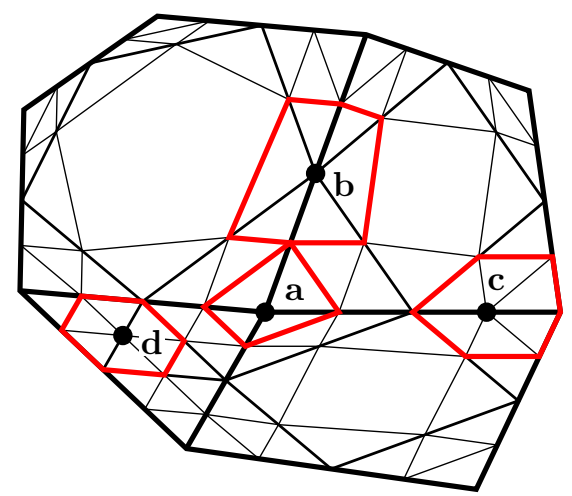

Figure 7: Vertex 1-rings of $\mathcal{P}_{i+2}^{\circ}$ projected into a plane that are contained in a vertex 1-ring of $\mathcal{P}_{i}^{\circ}$.

Similarly to the estimate in Equation 3.3 of Theorem 3.1, we observe that $s$-diam $R_{2}<s$-diam $R$ for any of these 1 -rings $R_{2}$ as long as diam $R>0$ and note that the reduction depends continuously on $R$. Note that this is also true if some vertices of $R$ coalesce. Hence for all possible $R$ not contained in an open spherical cap with spherical diameter $\alpha=$ maximum vertex angle of $\mathcal{P}_{1}$, the reduction factor stays below some $\gamma<1$ since a continuous function has a maximum over a compact set. Thus Equation (3.7) follows for all triangle vertex 1-rings of the set $T$ of all triangle vertex 1-rings not contained in a closed spherical cap with diameter $\alpha$. If $R$ does not lie in $T$, we consider its 
central projection, denoted by $\bar{R}$, into a plane as described above. Under this projection all ratios $\gamma \in\left[\varrho, \varrho^{-1}\right]$ on spherical line segments are projected into ratios in some interval $\left[\sigma, \sigma^{-1}\right] \subset(0, \infty)$ with $\sigma=\epsilon /(1-\epsilon) \in(0,1)$.

Using the notation of Figure 7, we observe that the following holds for the four 1-ring types in the plane where we pick $\mathbf{a}$ as the origin:

- The new 1-ring around $\mathbf{a}$ is contained in $\epsilon^{-2} \bar{R}$.

- The new 1-rings around $\mathbf{b}$ and $\mathbf{c}$ are contained in $\epsilon^{-2} \bar{R}$.

- The new 1-ring around $\mathbf{d}$ is contained in $\epsilon^{-1} \bar{R}$.

Consequently, the $p$-diameters shrink by at least the factor $\epsilon^{-1}$, which concludes the proof for Case 1 .

Case 2. Second, we consider a vertex 1-ring $R_{k}$ containing a non-triangular face $F_{k}$ of $\mathcal{P}_{i+k}^{\circ}$. Such a 1-ring lies in a face $F$ of $\mathcal{P}_{i}^{\circ}$ and is surrounded by triangles, see Figure 8. Its vertices are generated at three different refinement steps. The vertices of $F$ are said to be of generation 0 and the vertices generated from vertices of generation $j$ are referred to as vertices of generation $j+1$. Because of Remark 2.5, s-diam $F \leq$ maximum vertex angle $<\pi$. Hence $p$-diam $R_{k}=$ diam $\bar{R}_{k}$, where $\bar{R}_{k}$ (and $\overline{\bar{F}}$ ) denote the image(s) under the central projection introduced above.

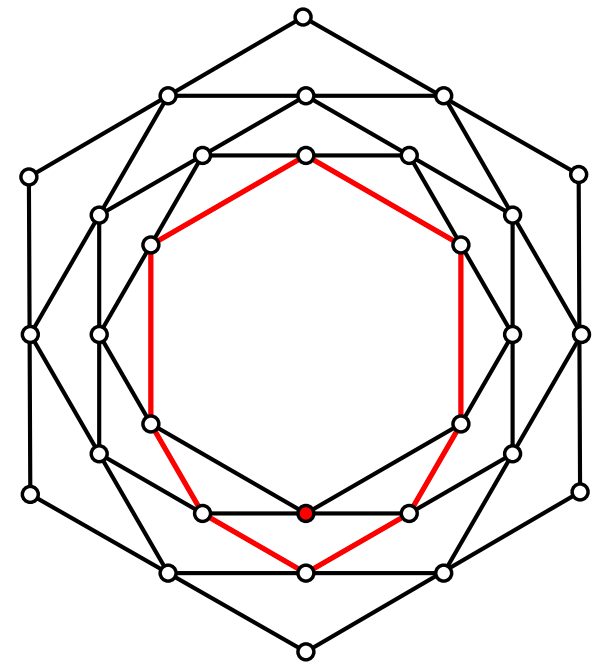

Figure 8: A non-triangular 1-ring (in red) with vertices of generation 2, 3, 4 inside an $(2 k-2)$ gon of generation 0 .

To show that $p$-diam $R_{k}<p$-diam $F$, we assume for a proof by contradiction that we had two points $\mathbf{p}$ and $\mathbf{q}$ in $\bar{R}_{k}$ with $\operatorname{dist}(\mathbf{p}, \mathbf{q})=\operatorname{diam} \bar{R}_{k}=\operatorname{diam} \bar{F}$ 
for $\operatorname{diam} F>0$. The points $\mathbf{p}$ and $\mathbf{q}$ are of some generations $p$ and $q$, i. e., they are determined by two groups $G_{\mathbf{p}}$ and $G_{\mathbf{q}}$ of $p+1$ and $q+1$ vertices of $\bar{F}$, respectively.

Since we are assuming $\operatorname{dist}(\mathbf{p}, \mathbf{q})=\operatorname{diam} \bar{F}>0$, both points lie on the boundary of $\bar{F}$ which implies that $G_{\mathbf{p}}$ and $G_{\mathbf{q}}$ consist of $p+1$ or $q+1$ equal vertices, respectively, and since diam $\bar{F}>0$, that they are disjoint. Hence $F$ had at least $p+q+2 \geq k+1+k-1=2 k$ vertices which contradicts the prerequisites of the theorem. (Although by definition, polyhedra do not have coalescing vertices, we obtain coalescing vertices in the limit for sequences of faces with diameter 1 in this proof below. Further note that if, e. g., p did not consist of equal vertices, p can lie on the boundary of $\bar{F}$ only if $G_{\mathbf{p}}$ spanned a non-degenerate edge. If in this case $G_{\mathbf{q}}$ and $G_{\mathbf{p}}$ were not disjoint, their union would span either an edge or a triangle depending on whether $G_{\mathbf{q}}$ spans a point or an edge. However $\mathbf{p}$ could not be a vertex of this edge or triangle meaning that $\operatorname{dist}(\mathbf{p}, \mathbf{q}) \neq \operatorname{diam} \bar{F}$. Hence $G_{\mathbf{p}}$ and $G_{\mathbf{q}}$ each do not span more than just a point.)

Consequently,

$$
q=\frac{\operatorname{diam} \bar{R}}{\operatorname{diam} \bar{F}}<1
$$

for all possible $R$ and $F$ with diam $F>0$.

Under the projection of the spherical dual honeycomb scheme restricted to $F$, the ratios in $\left[\varrho, \varrho^{-1}\right]$ are transformed into ratios in some interval $\left[\sigma, \sigma^{-1}\right] \subset$ $(0, \infty)$. Therefore we consider the quotient $q$ above for any dual honeycomb construction applied to $\bar{F}$ with ratios in $\left[\sigma, \sigma^{-1}\right]$. Since $q$ does not change if we scale $\bar{F}$ while keeping the construction ratios fixed, it suffices to restrict the quotient to the faces $\bar{F}$ with diameter 1 . As a continuous function attains a maximum over compact sets, $q$ is bounded by some constant $\lim _{i \rightarrow \infty} \mathbf{a}_{i}$ below 1.

\section{The corner pushing scheme}

We dualize the honeycomb scheme and call the dual scheme the corner pushing scheme or just the pushing scheme and denote it by P. Under a pushing scheme, a polyhedron $\mathcal{P}$ is refined as follows: On each edge a point is chosen dividing the edge such that the ratio lies between $\varrho$ and $\varrho^{-1}$, where $\varrho=\epsilon$ : $(1-\epsilon)>0, \epsilon \in(0,1 / 2]$, is the pushing tolerance. The new vertices on any two adjacent edges are connected by $f$-edges that split every face of valence $v$ into $v$ triangles and a smaller face of valence $v$ surrounded by the triangles. Finally, the triangles are rotated around their $f$-edges into the old polyhedron by moving the old vertices towards the origin to any positions where convexity of the thus transformed polyhedron is still preserved as shown in Figure 9. 


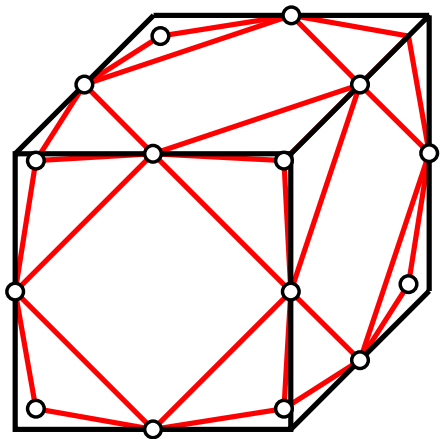

Figure 9: The pushing scheme applied to a cube.

Figure 10 shows pushing surfaces generated from a cuboid and an icosahedron with $\varrho=1 / 2$ and different pushing percentages $\tau$ defining to which percentage vertices are moved towards their extreme positions.

The points picked on the edges correspond to the planes chosen in the honeycomb scheme on every edge. The origin is dual to the ideal plane and moving a point to the origin means in the primal space to translate the dual plane away from the origin. Dualizing Theorem 3.4, we obtain

4.1 Theorem (No line segments by P). The limiting surfaces generated by the pushing scheme have no line segments.

Dualizing the example in Section 1.3 shows that the pushing scheme generates surfaces with corners in general. However, we can easily prove that it does not increase the 1-rings in the Gaussian images:

4.2 Theorem (No larger face angles under $\mathrm{P})$. Let $\left(\mathcal{P}_{i}\right)_{i \in \mathbb{N}}$ be a pushing sequence. Let $\mu_{i}$ be the maximum face angle of $\mathcal{P}_{i}$, then $\mu_{i} \geq \mu_{i+1}$ for all $i$.

Proof. The Gaussian images $\mathcal{P}_{i}^{\circ}$ have the same connectivity as some polyhedra generated by the honeycomb scheme and any vertex 1-ring of $\mathcal{P}_{i+1}^{\circ}$ is contained in some vertex 1-ring of $\mathcal{P}_{i}^{\circ}$ as illustrated in Figure 11.

4.3 Remark. The pushing scheme resembles Tóth's construction of the kernel, see $[21,11]$. There $\mathcal{P}_{n+1}$ is the convex hull of the edge midpoints of $\mathcal{P}_{n}$.

\section{Mixed honeycomb pushing refinement}

Our goal is to combine the corner pushing with the honeycomb scheme so as to obtain a self-dual class of schemes generating $C^{1}$ surfaces without line seg- 


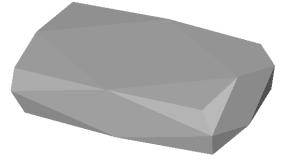

$\mathrm{P}_{20}^{1}$

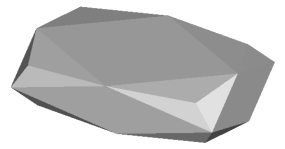

$\mathrm{P}_{50}^{1}$

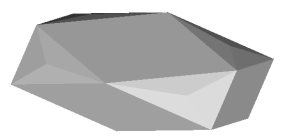

$\mathrm{P}_{90}^{1}$

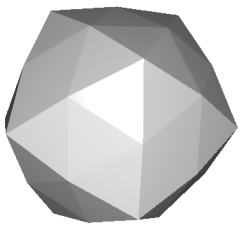

$\mathrm{P}_{20}^{1}$

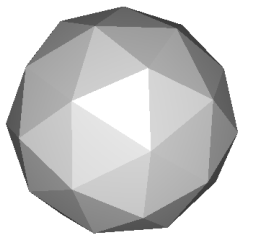

$\mathrm{P}_{50}^{1}$

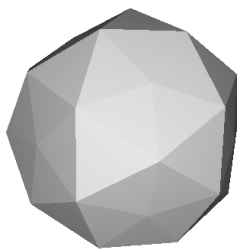

$\mathrm{P}_{90}^{1}$

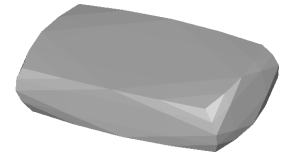

$\mathrm{P}_{20}^{2}$

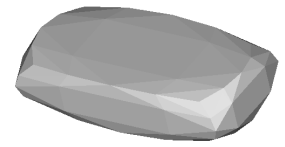

$\mathrm{P}_{50}^{2}$

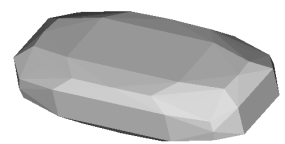

$\mathrm{P}_{90}^{2}$

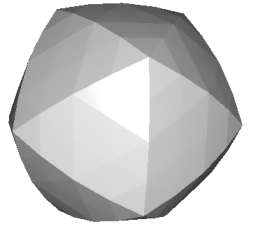

$\mathrm{P}_{20}^{2}$

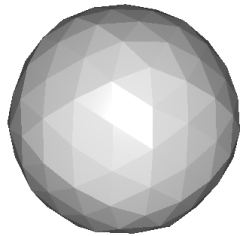

$\mathrm{P}_{50}^{2}$

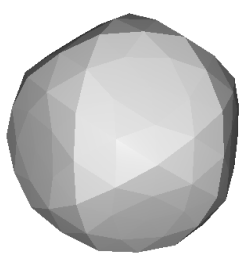

$\mathrm{P}_{90}^{2}$

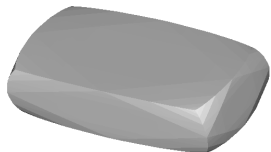

$\mathrm{P}_{20}^{7}$

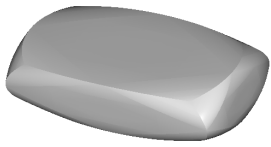

$P_{50}^{7}$

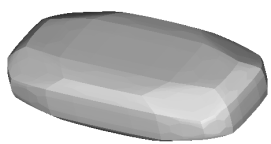

$\mathrm{P}_{90}^{7}$

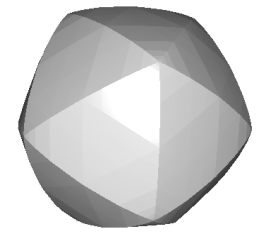

$\mathrm{P}_{20}^{7}$

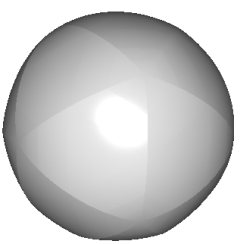

$\mathrm{P}_{50}^{7}$

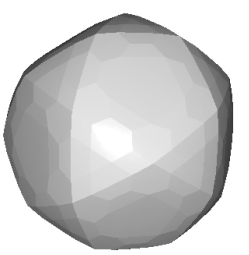

$\mathrm{P}_{90}^{7}$

Figure 10: The cuboid and icosahedron shown in Figure 2 refined by 1, 2 and 7 iterations of the pushing scheme with offsetting percentages 20,50 and 90 from top to bottom. 


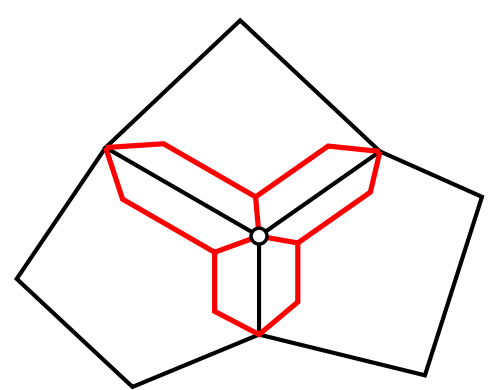

(a)

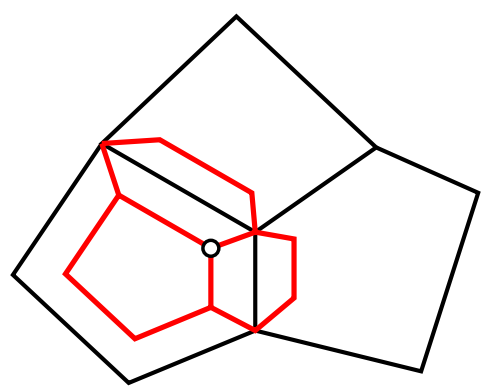

(b)

Figure 11: Vertex 1-rings generated by a honeycomb step.

ments. Therefore we define mixed honeycomb pushing refinement or MHPschemes as the schemes where we repeatedly apply $h$ honeycomb steps followed or preceeded by $p$ pushing steps to a polyhedron $\mathcal{P}_{1}$. Hence, an MHP scheme is dual to an MHP scheme. We call a sequence of polyhedra $\left(\mathcal{P}_{i}\right)_{i \in \mathbb{N}}$ generated by an MHP scheme an MHP sequence and accesible if in addition

maximum vertex valence $<2 h$

and

maximum face valence $<2 p$.

We note that both pushing and the honeycomb scheme preserve vertex valences and create new vertices with valence 3 . Hence an MHP scheme preserves vertex valences and generates vertices of valence 3 and 6 . Dually, an MHP scheme also preserves face valences and generates faces of valence 6 and 3. Hence for any MHP sequence $\left(\mathcal{P}_{i}\right)_{i \in \mathbb{N}}$, no $\mathcal{P}_{i}$ has greater vertex and face valences than the first $\mathcal{P}_{j}$ generated by applying at least one honeycomb and one pushing operation.

Honeycomb sequences are bounded and the same holds for MHP sequences. Here we prove the dual statement:

5.1 Theorem (Non-empty interior of MHP-limits). Every MHP sequence $\left(\mathcal{P}_{i}\right)_{i \in \mathbb{N}}$ has a non-empty interior, i.e., there is a ball around the origin $\mathbf{o}$ contained in all polyhedra $P_{i}$.

Proof. We call a vertex $\mathbf{v}$ of $\mathcal{P}_{i}$ old if it equals or if it is obtained by pushing a vertex $\mathbf{u}$ of $\mathcal{P}_{i-1}$. Calling such vertices $\mathbf{u}$ and $\mathbf{v}$ of $\mathcal{P}_{i-1}$ and $\mathcal{P}_{i}$ associated, let $\left(\mathbf{v}_{i}\right)_{i \in \mathbb{N}}$ be a sequence of associated vertices where $\mathbf{v}_{i}$ is a vertex of $\mathcal{P}_{i}$. Further let $\mathcal{C}_{i}$ be the convex hull of the new, i. e., not old vertices in $\mathcal{P}_{i}$. Let $\mathcal{B}_{i}$ be the union of the faces of $\mathcal{C}_{i}$ whose vertices are all neighbors of $\mathbf{v}_{i}$ with respect to $\mathcal{P}_{i}$. Hence if we cut $\mathcal{P}_{i}$ along all $\mathcal{B}_{i}$, we obtain $\mathcal{C}_{i}$, i. e., the cones with bases $\mathcal{B}_{i}$ and vertices $\mathbf{v}_{i}$ are what we cut off from $\mathcal{P}_{i}$ to obtain $\mathcal{C}_{i}$. In direction $\mathbf{v}_{i}$ then, $\mathcal{B}_{i+1}$ lies completely behind $\mathcal{B}_{i}$. 
Hence, for any vertex $\mathbf{v}$ of $\mathcal{P}_{1}$, the line segment $\left[\mathbb{R}^{3} \mathbf{v} \cap \mathcal{B}_{1}, \mathbb{R}^{3} \mathbf{v} \cap \mathcal{B}_{2}\right]$ lies in all $\mathcal{P}_{i}$. Since the vertices of $\mathcal{P}_{1}$ span $\mathbb{R}^{3}$ by Assumption 2.1, we see that the convex hull of the line segments above has a non-empty interior which proves the theorem.

Since any MHP sequence is dual to an MHP sequence and since a polyhedron with $\mathbf{o}$ in its interior is dual to a bounded polyhedron, Theorem 5.1 dualizes to

5.2 Theorem. Any MHP sequence $\left(\mathcal{P}_{i}\right)_{i \in \mathbb{N}}$ is bounded.

5.3 Corollary (A bound for the vertex angles of an MHP sequence). All vertex angles of an MHP sequence $\left(\mathcal{P}_{i}\right)_{i \in \mathbb{N}}$ are smaller than some $\alpha<180^{\circ}$.

Proof. According to Theorems 5.1 and 5.2, we can find concentric balls $\mathcal{B}_{r}$ and $\mathcal{B}_{d}$ of radius $r$ and $d$ such that $\mathcal{B}_{r} \subset \mathcal{P}_{i} \subset \mathcal{B}_{d}$ for all $i$. Hence all vertex angles $\varphi$ of $\mathcal{P}_{i}$ satisfy

$$
\varphi \leq \alpha:=2 \arccos \frac{r}{d}<180^{\circ}
$$

see Figure 12 .

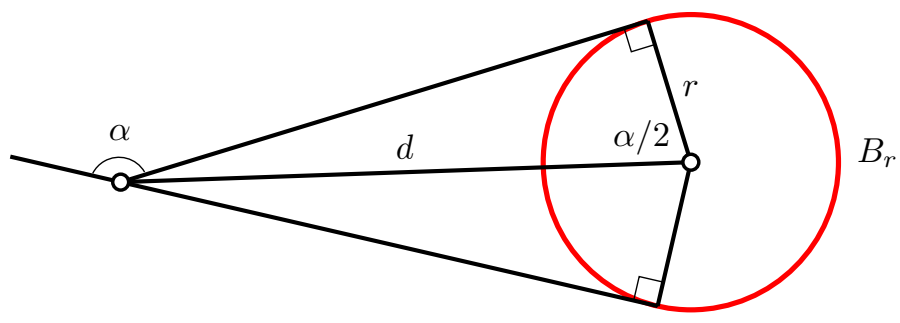

Figure 12: Estimating the angles.

Corollary 5.3 can be used to replace Remark 2.5 in the proof of Theorem 3.6. Hence using Remark 3.5 we can combine Theorems 3.6 and 4.2 to arrive at

5.4 Theorem (MHP flattens all face angles). Let $\left(\mathcal{P}_{i}\right)_{i \in \mathbb{N}}$ be an accessible MHP sequence. Let $\mu_{i}$ be the maximum face angle of $\mathcal{P}_{i}$, then there are constants $c>0$ and $\gamma \in(0,1)$ such that $\mu_{i}<c \cdot \gamma^{i}$.

Dualizing Theorem 5.4, we get in view of Remark 2.6

5.5 Theorem (MHP shrinks edges geometrically). Let $\left(\mathcal{P}_{i}\right)_{i \in \mathbb{N}}$ be an accessible MHP sequence and let $\mu_{i}$ be the maximum 1-ring diameter of $\mathcal{P}_{i}$, then there are constants $c>0$ and $\gamma \in(0,1)$ such that $\mu_{i}<c \cdot \gamma^{i}$.

5.6 Theorem. Any MHP sequence $\left(\mathcal{P}_{i}\right)_{i \in \mathbb{N}}$ converges to a (convex) polyhedron $L$. 
Proof in a nutshell. Any vertex is pushed towards the origin and converges to a limiting vertex. The limiting vertices belonging to the vertices of $\mathcal{P}_{i}$ span a polyhedron $\mathcal{L}_{i}$ lying inside $\mathcal{P}_{i}$ and $\mathcal{L}_{i+1}$. Due to Theorem 5.2, the nested sequence $\left(\mathcal{L}_{i}\right)_{i \in \mathbb{N}}$ is bounded and thus converges. Since the pushing distances are bounded by twice the maximum edge length which shrinks geometrically because of Theorem $5.5, \operatorname{dist}\left(\mathcal{P}_{i}, \mathcal{L}_{i}\right) \rightarrow 0$, which concludes the proof.

Proof. The honeycomb scheme preserves the vertices and the pushing scheme pushes the vertices inwards. Hence any vertex $\mathbf{p}$ of any $\mathcal{P}_{i}$ is pushed to or equals a vertex $\mathbf{p}_{j}$ in $\mathcal{P}_{j}$ for all $j \geq i$. First we prove that a sequence $\left(\mathbf{p}_{j}\right)_{j=i}^{\infty}$ converges geometrically. From Theorem 5.5, we know that the maximum edge length in $\mathcal{P}_{i}$ is bounded by some $c \gamma^{i}$, where $\gamma \in(0,1)$ and $c$ are constants.

If $\mathbf{p}_{i} \neq \mathbf{p}_{i+1}$, i. e., if $\mathbf{p}_{i+1}$ is generated by the pushing scheme, then $\mathbf{p}_{i+1}$ is connected to $\mathbf{p}_{i}$ via an edge in $\mathcal{P}_{i+1}$ and a part of an edge in $\mathcal{P}_{i}$, see Figure 13. Hence

$$
\left\|\mathbf{p}_{i}-\mathbf{p}_{i+1}\right\|<c \gamma^{i}+c \gamma^{i+1} \leq 2 c \gamma^{i} .
$$

This means that the $\mathbf{p}_{i}$ converge to some point $\mathbf{p}_{\infty}$, where

$$
\left\|\mathbf{p}_{i}-\mathbf{p}_{\infty}\right\| \leq \sum_{j=i}^{\infty} 2 c \gamma^{j}=\frac{2 c}{1-\gamma} \gamma^{i}
$$

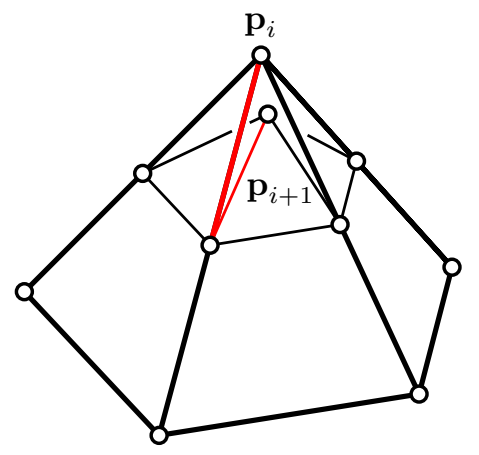

Figure 13: A step of the pushing scheme illustrated for two associated 1-rings.

The limiting points $\mathbf{p}_{\infty}$ for all vertices $\mathbf{p}$ of $\mathcal{P}_{i}$ form the vertices of some convex polyhedron $\mathcal{L}_{i}$, which is the limiting polyhedron of the polyhedra $\mathcal{K}_{j}:=$ convex hull $\left\{\mathbf{p}_{j} \mid \mathbf{p}\right.$ a vertex of $\left.\mathcal{P}_{i}\right\}, j \geq i$. To see that $\mathcal{L}_{i}$ is convex, note that the vertices of $\mathcal{K}_{j}$ are also vertices of $\mathcal{P}_{j}$ and that the $\mathbf{p}_{j}$ move towards the origin. Hence the $\mathcal{K}_{j}$ are convex and nested, i. e., $\mathcal{K}_{j} \supset \mathcal{K}_{j+1}$. Thus $\mathcal{L}_{i}=\bigcap_{j=i}^{\infty} \mathcal{K}_{j}$ is convex as it is the intersection of convex sets, see Figure 14 for a two-dimensional illustration. 


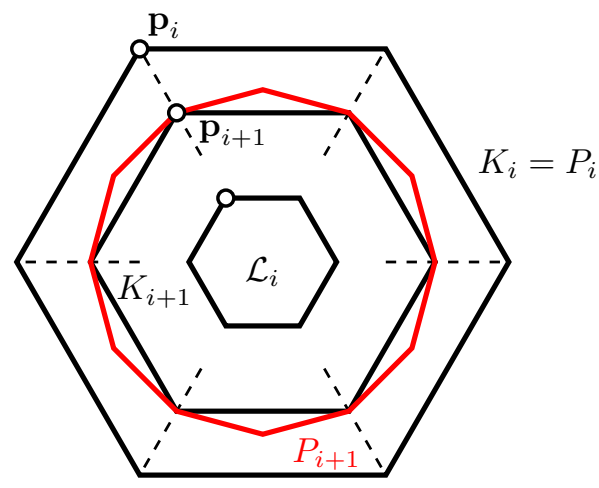

Figure 14: The polyhedra $\mathcal{P}_{i}, \mathcal{P}_{i+1}, K_{i+1}$ and $\mathcal{L}_{i}$ illustrated schematically by planar polygons.

Further note that the vertices of $\mathcal{L}_{i}$ are also vertices of any $\mathcal{L}_{j}, j \geq i$. Hence the sequence $\left(\mathcal{L}_{j}\right)_{j=0}^{\infty}$ is monotonously increasing. Because of Theorem 5.2, it is also bounded and we conclude that the $\mathcal{L}_{j}$ converge to a limiting polyhedron $\mathcal{L}$ containing all vertices of every $\mathcal{L}_{i}$. Showing that $\mathcal{L}$ is also the limit of the sequence $\left(\mathcal{P}_{i}\right)$ will finish the proof: The polyhedra $\mathcal{P}_{i}$ and $\mathcal{L}_{i}$ are topologically equivalent and if $\mathbf{v}$ denotes a vertex of $\mathcal{P}_{i}$, we denote its corresponding vertex in $\mathcal{L}_{i}$ by $\mathbf{v}^{\prime}$. Any point $\mathbf{p}$ on $\mathcal{P}_{i}$ lies in a face of $\mathcal{P}_{i}$ and is a convex combination of its vertices $\mathbf{v}_{1}, \ldots, \mathbf{v}_{k}$, i.e.,

$$
\mathbf{p}=\sum_{r=1}^{k} \mathbf{v}_{r} \nu_{r}, \text { where } \sum_{r=1}^{k} \nu_{r}=1 \text { and } \nu_{r} \geq 0 .
$$

We compare $\mathbf{p}$ with the point

$$
\mathbf{p}^{\prime}=\sum_{r=1}^{k} \mathbf{v}_{r}^{\prime} \nu_{r}
$$

in $\mathcal{L}_{i}$ and obtain

$$
\begin{aligned}
\left\|\mathbf{p}^{\prime}-\mathbf{p}\right\| & =\left\|\sum\left(\mathbf{v}_{r}^{\prime}-\mathbf{v}_{r}\right) \nu_{r}\right\| \\
& \leq \sum\left\|\mathbf{v}_{r}^{\prime}-\mathbf{v}_{r}\right\| \nu_{r} \\
& =\sum \frac{2 c}{1-\gamma} \gamma^{i} \nu^{r} \\
& =\frac{2 c}{1-\gamma} \gamma^{i} .
\end{aligned}
$$

Thus

$$
\operatorname{dist}\left(\mathcal{P}_{i}, \mathcal{L}_{i}\right) \leq \frac{2 c}{1-\gamma} \gamma^{i}
$$

which implies that the $\mathcal{P}_{i}$ converge to $\mathcal{L}$. 
Finally, we show that the limit $\mathcal{P}_{\infty}$ of any MHP sequence $\left(\mathcal{P}_{i}\right)_{i=0}^{\infty}$ is a $\mathcal{C}^{1}$ surface, which means to show that $\mathcal{P}_{\infty}$ has a continuous normal field:

First we consider the normals of $\mathcal{P}_{\infty}$ at the limiting points $\mathbf{p}_{\infty}$ introduced in the proof of Theorem 5.6, see Equation (5.7). These points form the limiting vertex set $\mathcal{V}:=\left\{\mathbf{p}_{\infty} \mid \mathbf{p}\right.$ is a vertex of $\left.\mathcal{P}_{i}, i \in \mathbb{N}\right\}$ which is dense in $\mathcal{P}_{\infty}$ because of Theorem 5.5. Any point $\mathbf{p}_{\infty}$ is the limit of a sequence $\left(\mathbf{p}_{j}\right)_{j>i}$ and the Gaussian images of the 1-rings around the $\mathbf{p}_{j}$ form a nested sequence of spherical polygons shrinking to a unit vector $\mathbf{n}_{\mathbf{p}}$ which is a normal vector of $\mathcal{P}_{j}$ at $\mathbf{p}_{j}$ for all $j \geq i$.

Because of Theorem 5.4, the discrete normal field $\mathbf{n}_{\mathbf{p}}, \mathbf{p} \in \mathcal{V}$, is dense in $S^{2}$. Due to Theorem 5.5, this then rules out corners with a 2-parameter family of tangent planes on $\mathcal{P}_{\infty}$ but does not prohibit pinch points, i. e., point with a 1- but not a 2-parameter family of tangent planes. For the latter, we need to show that points in any infinitesimally small neighborbood have the same normal, i. e., we need to show that the discrete normal field can be extended to a continuous normal field of $\mathcal{P}_{\infty}$. Therefore we partition $\mathcal{P}_{\infty}$ into patches forming neighborhoods on $\mathcal{P}_{\infty}$ controlled by face 6 -rings of the $\mathcal{P}_{i}$.

5.8 Definition. Let $\pi$ be the central projection $\mathbb{R}^{3} \backslash\{\mathbf{o}\} \rightarrow \mathcal{P}_{\infty}$ with the projection center $\mathbf{o}$. Then for all faces $F$ of any $\mathcal{P}_{i}$, we call $\pi F$ an $i$-th generation patch of $\mathcal{P}_{\infty}$.

5.9 Lemma. Let $F$ be a face of any $\mathcal{P}_{i}$. Then the patch $\pi F$ and the normals over $\mathcal{V} \cap \pi F$ are determined by the 6 -ring $R_{6} F$ of faces around $F$. Moreover, $\pi R_{6} F \supset \pi F$ and $\left(R_{6} F\right)^{\circ} \supset\left\{\mathbf{n}_{\mathbf{p}} \mid \mathbf{p} \in \mathcal{V} \cap \pi F\right\}$, where here $\left(R_{6} F\right)^{\circ}$, different from Definition 2.2, is not just a mesh but also contains all points of $S^{2}$ lying in a face of this mesh.

Proof in a nutshell. Similarly to other refinement schemes, $\mathrm{H}$ and $\mathrm{P}$ generate more faces while they shrink an open mesh $\mathcal{M}$ with a boundary. Analyzing how large we have to choose $\mathcal{M}$ to balance the gains and losses along the boundary, we obtain the lemma.

Proof. Let $\mathcal{M}$ and $\mathcal{N}$ be submeshes of $\mathcal{P}_{i}$ and $\mathcal{P}_{j}$, respectively. Denote the mesh $\mathcal{N}$ without its $r$ outmost layers of faces by $W_{r} \mathcal{N}$ and denote the relation $\pi \mathcal{M} \supset \pi \mathcal{N}$ simply by $\mathcal{M} \sqsupset \mathcal{N}$. Let $\mathrm{H} \mathcal{M}$ and $\mathrm{P} \mathcal{M}$ be the meshes obtained from $\mathcal{M}$ by the honeycomb or pushing scheme, respectively.

Since $\pi \mathbf{p}_{j}=\mathbf{p}_{\infty}$ for all vertices $\mathbf{p}$ of any $\mathcal{P}_{i}$ and all $j \geq i$, we obtain - leaving the verification to the reader - for all $q, r, k \in \mathbb{N}$

$$
\begin{aligned}
& \mathrm{H}^{q} \mathcal{M} \sqsupset W_{2} \mathcal{M}, \\
& \mathrm{P}^{r} \mathcal{M} \sqsupset W_{1} \mathcal{M},
\end{aligned}
$$


and for $S=\mathrm{H}^{q} \mathrm{P}^{r}$ or $S=\mathrm{P}^{r} \mathrm{H}^{q}$

$$
S \mathcal{M} \sqsupset W_{3} \mathcal{M}
$$

as well as

$$
W_{2 k} S \mathcal{M} \sqsupset W_{3+k} \mathcal{M}
$$

This implies

$$
W_{6} S \mathcal{M} \sqsupset W_{6} \mathcal{M}
$$

and

$$
W_{6} S^{k} \mathcal{M} \sqsupset W_{6} S^{k-1} \mathcal{M} \sqsupset \cdots \sqsupset W_{6} \mathcal{M},
$$

which shows that $\mathcal{M}=R_{6} F$ determines $\pi F$ and its normals over $\mathcal{V} \cap \pi F$.

Lemma 5.9 in conjunction with Theorems 5.4 and 5.5 shows that every point q on $\mathcal{P}_{\infty}$ has neighborhoods $U_{i}$ consisting of a vertex 1-ring of $i$-th generation patches with arbitrarily small Gaussian images. Although we do not need it, we note that the $U_{i}$ become arbitrarily small, i. e., $U_{i} \rightarrow\{\mathbf{q}\}$, due to Theorem 5.5. Moreover, Theorem 5.4 shows that the normal vectors $\mathbf{n}_{\mathbf{p}}, \mathbf{p} \in \mathcal{V} \cap U_{i}$, converge uniformly for all $\mathbf{q}$ to a single vector $\mathbf{n}_{\mathbf{q}}$. Together with Theorem 5.6, this then proves the main theorem:

5.10 Theorem (MHP surfaces are smooth). Any accessible MHP sequence converges to a $C^{1}$ surface without line segments.

Proof in a nutshell. Due to Theorems 5.5 and 5.4, the limiting vertex set $\mathcal{V}$ is dense on $\mathcal{P}_{\infty}$ and has a unique normal field. Because of Lemma 5.9 this normal field can be extended continuously to all of $\mathcal{P}_{\infty}$ which shows the theorem.

\section{Test examples}

We tested the MHP scheme with different offsetting and pushing parameters and show some results in Figure 15 for a cuboid and an icosahedron as initial polyhedra, where we alternatingly apply 4 honeycomb and 4 pushing steps to polyhedra with valences $\leq 6$. The results resemble much the surfaces that we get by the pure honeycomb or pushing scheme depending on the first iteration steps of the MHP scheme, see Figures 2 and 10.

In an attempt to obtain rounder surfaces, we also alternatingly applied single or double honeycomb and pushing steps as shown in Figure 16. However, in all our experiments on these and other polyhedra, the limiting surfaces always 


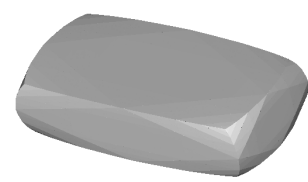

$\mathrm{H}_{20}^{4} \mathrm{P}_{20}^{4}$

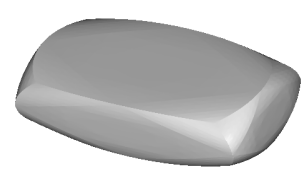

$\mathrm{H}_{0.5}^{4} \mathrm{P}_{0.5}^{4}$

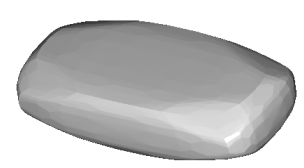

$\mathrm{H}_{80}^{4} \mathrm{P}_{80}^{4}$

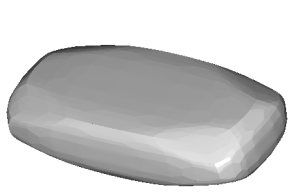

$\mathrm{H}_{20}^{4} \mathrm{P}_{80}^{4}$

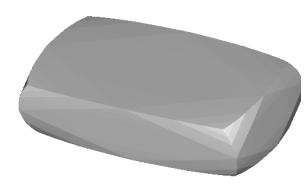

$\mathrm{H}_{80}^{4} \mathrm{P}_{20}^{4}$

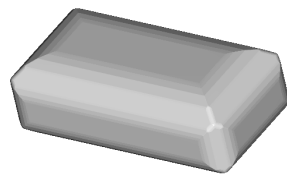

$\mathrm{P}_{20}^{4} \mathrm{H}_{20}^{4}$

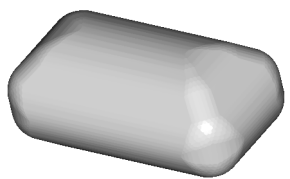

$\mathrm{P}_{0.5}^{4} \mathrm{H}_{0.5}^{4}$
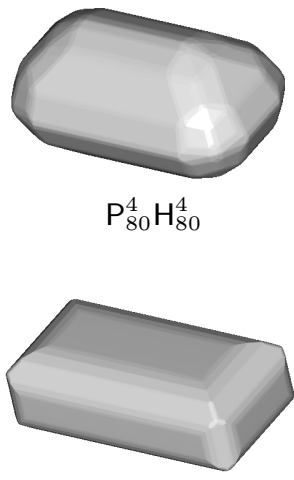

$\mathrm{P}_{80}^{4} \mathrm{H}_{20}^{4}$

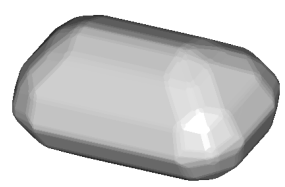

$\mathrm{P}_{20}^{4} \mathrm{H}_{80}^{4}$

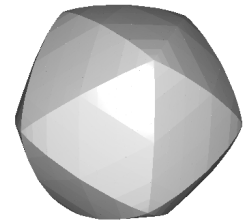

$\mathrm{H}_{20}^{4} \mathrm{P}_{20}^{4}$

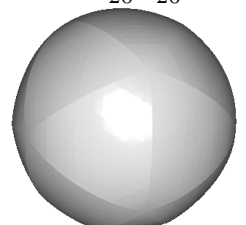

$\mathrm{H}_{50}^{4} \mathrm{P}_{50}^{4}$

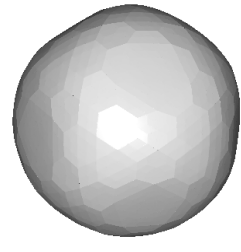

$\mathrm{H}_{80}^{4} \mathrm{P}_{80}^{4}$

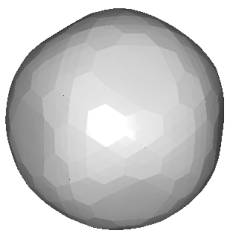

$\mathrm{H}_{20}^{4} \mathrm{P}_{80}^{4}$

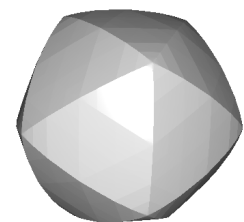

$\mathrm{H}_{80}^{4} \mathrm{P}_{20}^{4}$

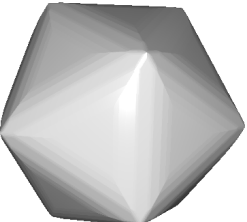

$\mathrm{P}_{20}^{4} \mathrm{H}_{20}^{4}$

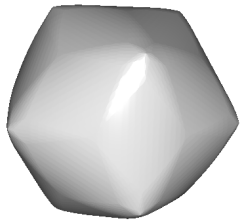

$\mathrm{P}_{50}^{4} \mathrm{H}_{50}^{4}$

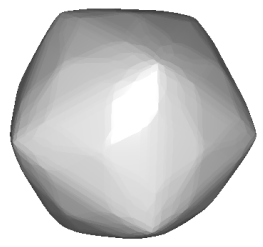

$\mathrm{P}_{80}^{4} \mathrm{H}_{80}^{4}$

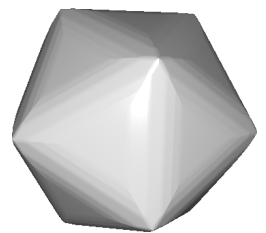

$\mathrm{P}_{80}^{4} \mathrm{H}_{20}^{4}$

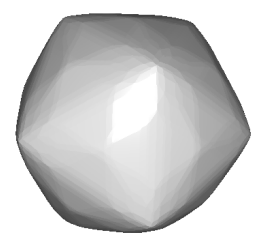

$\mathrm{P}_{20}^{4} \mathrm{H}_{80}^{4}$

Figure 15: Polyhedra generated by $\mathrm{H}^{4} \mathrm{P}^{4}$ and $\mathrm{P}^{4} \mathrm{H}^{4}$ from a cuboid and an icosahedron with pushing and honeycomb offsetting percentages $20 / 20,50 / 50,80 / 80,20 / 80,80 / 20$ from top to bottom. 


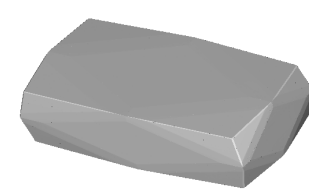

$\left(\mathrm{H}_{20} \mathrm{P}_{20}\right)^{4}$

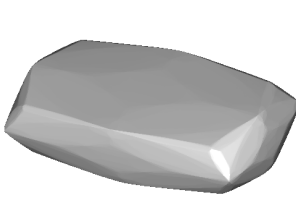

$\left(\mathrm{H}_{50} \mathrm{P}_{50}\right)^{4}$

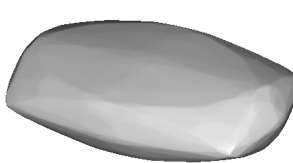

$\left(\mathrm{H}_{80} \mathrm{P}_{80}\right)^{4}$

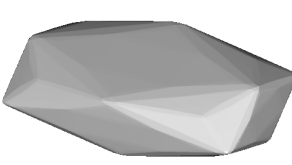

$\left(\mathrm{H}_{20} \mathrm{P}_{80}\right)^{4}$

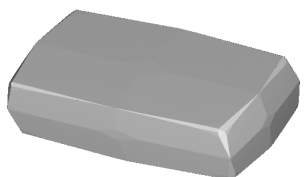

$\left(\mathrm{H}_{80} \mathrm{P}_{20}\right)^{4}$

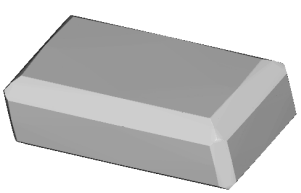

$\left(\mathrm{P}_{20} \mathrm{H}_{20}\right)^{4}$

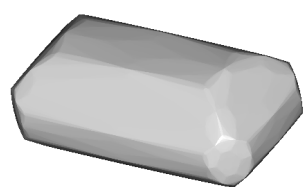

$\left(\mathrm{P}_{50} \mathrm{H}_{50}\right)^{4}$

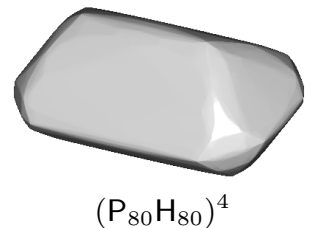

$\left(\mathrm{P}_{80} \mathrm{H}_{80}\right)^{4}$

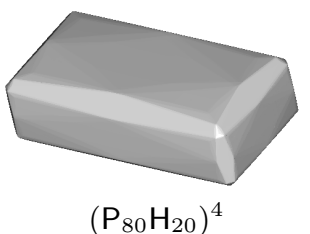

$$
\left(\mathrm{P}_{80} \mathrm{H}_{20}\right)^{4}
$$

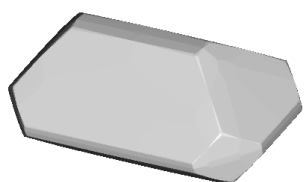

$\left(\mathrm{P}_{20} \mathrm{H}_{80}\right)^{4}$

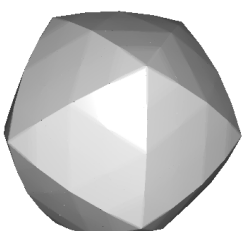

$\left(\mathrm{H}_{20}^{2} \mathrm{P}_{20}^{2}\right)^{2}$

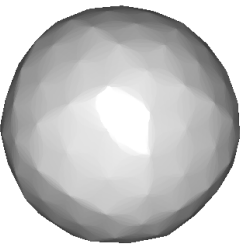

$\left(\mathrm{H}_{50}^{2} \mathrm{P}_{50}^{2}\right)^{2}$

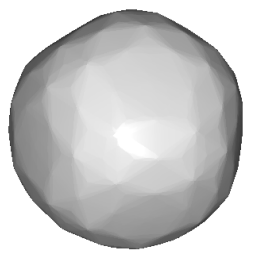

$\left(\mathrm{H}_{80}^{2} \mathrm{P}_{80}^{2}\right)^{2}$

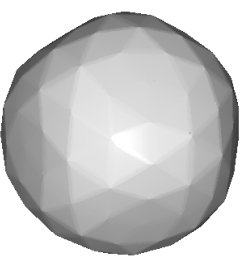

$\left(\mathrm{H}_{20}^{2} \mathrm{P}_{80}^{2}\right)^{2}$

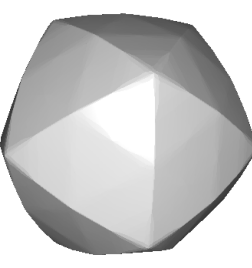

$\left(\mathrm{H}_{80}^{2} \mathrm{P}_{20}^{2}\right)^{2}$

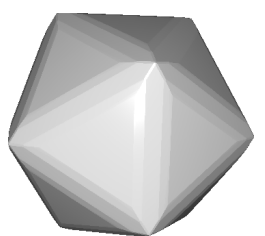

$\left(\mathrm{P}_{20}^{2} \mathrm{H}_{20}^{2}\right)^{2}$

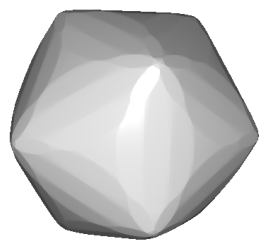

$\left(\mathrm{P}_{50}^{2} \mathrm{H}_{50}^{2}\right)^{2}$

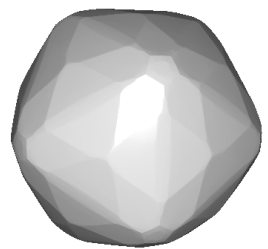

$\left(\mathrm{P}_{80}^{2} \mathrm{H}_{80}^{2}\right)^{2}$

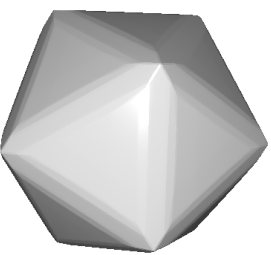

$\left(\mathrm{P}_{80}^{2} \mathrm{H}_{20}^{2}\right)^{2}$

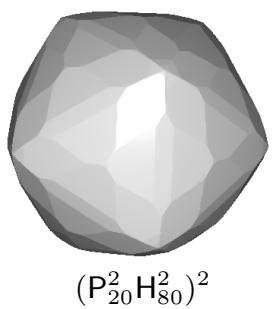

Figure 16: Polyhedra generated by $(\mathrm{HP})^{4},(\mathrm{PH})^{4},\left(\mathrm{H}^{2} \mathrm{P}^{2}\right)^{2}$ and $\left(\mathrm{P}^{2} \mathrm{H}^{2}\right)^{2}$ from a cuboid and icosahedron with pushing and honeycomb offsetting percentages 20/20,50/50, 80/80, 20/80, $80 / 20$ from top to bottom. 
take on a somewhat rounded polyhedral form. This is due to the different local convergence rates that depend on the vertex and face valences.

Most convex surfaces are not in $C^{2}$, see [11] and references cited in it. In particular, honeycomb surfaces are not $C^{2}$ as they tend to have planar segments almost everywhere. Pushing surfaces are even not $C^{1}$ as they have corners almost everywhere. Therefore, we cannot expect MHP surfaces to be very smooth either. Still we tried to compare the quality of the surfaces generated by the different schemes using reflection lines and discrete mean curvature visualization in Figure 17. Although the shaded images of the surfaces generated by applying the honeycomb scheme seven and eight times are not distinguishable, their curvature plots are quite different revealing a high sensitivity of the discrete curvature plots obtained by MeshLab with respect to corners, edges and the triangulation of the planar mostly hexagonal faces.

\section{Conclusion}

As we have shown, it is possible to modify the honeycomb scheme so as to get rid of the line segments in the limiting surfaces. However, practical tests show that the limiting surfaces suffer from a non-uniform convergence behavior due to different face and vertex valencies. Different from subdivision schemes with a finite number of extraordinary faces and vertices, MHP-schemes generate infinitely many faces and vertices both of valence 3 and 6 which are either extraordinary for the honeycomb or pushing steps. Nevertheless, the theoretical work has been beneficial as it helped to understand corner cutting for surfaces and to develop corner cutting schemes such as the $4-8, \sqrt{3}$ and the honeycomb cutting schemes which generate much better surfaces and can also generate spheres, see [3].

\section{Acknowledgement}

We thank Yijun $\mathrm{Xu}$ for implementing the scheme, testing several modifications of it and generating Figures 2, 10 and 15-17. The first author gratefully received financial support both from Hangzhou Dianzi University and Karlsruhe Institute of Technology.

\section{References}

[1] Akleman E., Srinivasan V.: Honeycomb Subdivision. In Ilyas Cicekli et al. (eds.) Proc. of The 17th International Symposium on Computer and Information Sciences. pp 137-141 Oct. 28-30, 2002.

[2] Barendrecht P., Sabin M., Kosinka J.: A bivariate C1 subdivision scheme based on cubic half-box splines. Computer Aided Geometric Design, Volume 71, 77-89 (2019). 


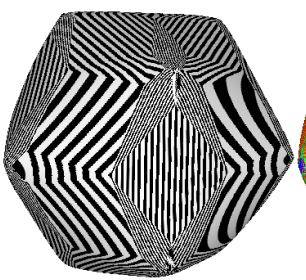

$\mathrm{H}^{7}$

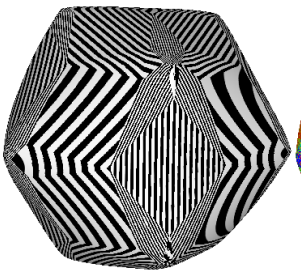

$\mathrm{H}^{8}$

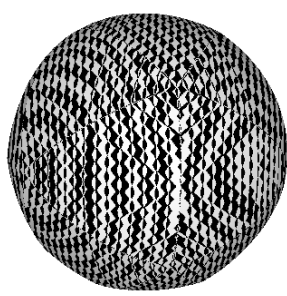

$\mathrm{H}^{4} \mathrm{P}^{4}$

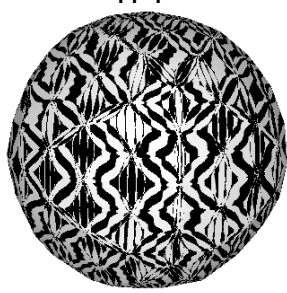

$\mathrm{H}^{2} \mathrm{P}^{2} \mathrm{H}^{2} \mathrm{P}^{2}$

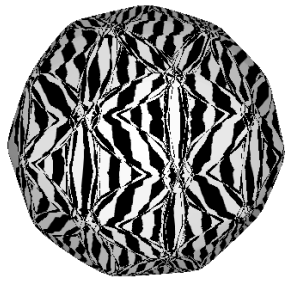

HPHPHPHP

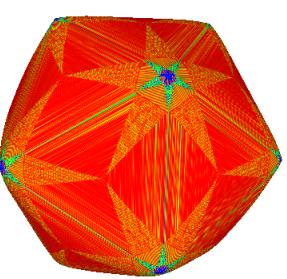

$\mathrm{H}^{7}$

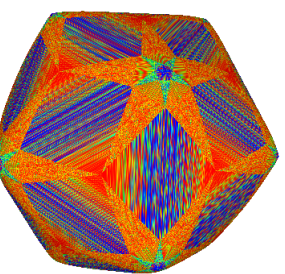

$\mathrm{H}^{8}$

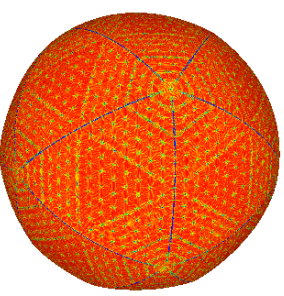

$\mathrm{H}^{4} \mathrm{P}^{4}$

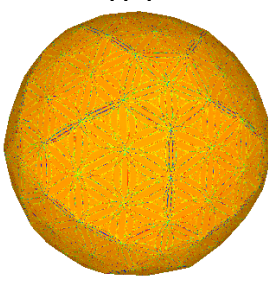

$\mathrm{H}^{2} \mathrm{P}^{2} \mathrm{H}^{2} \mathrm{P}^{2}$

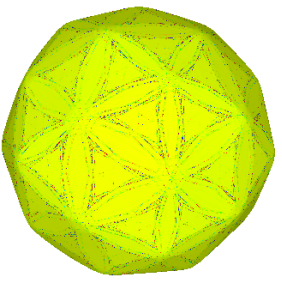

HPHPHPHP

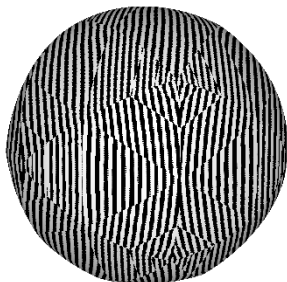

$\mathrm{P}^{7}$

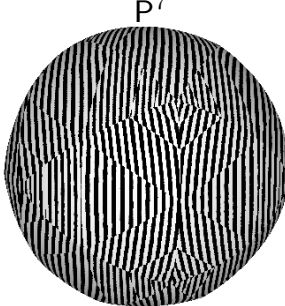

$\mathrm{P}^{8}$

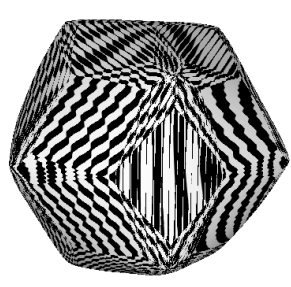

$\mathrm{P}^{4} \mathrm{H}^{4}$

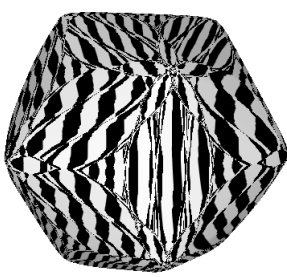

$\mathrm{P}^{2} \mathrm{H}^{2} \mathrm{P}^{2} \mathrm{H}^{2}$

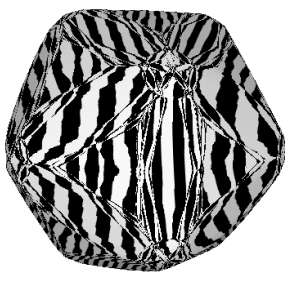

PHPHPHPH

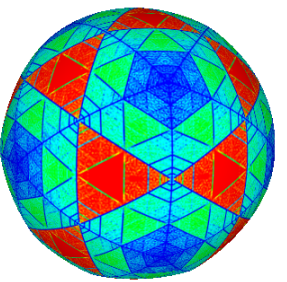

$\mathrm{P}^{7}$

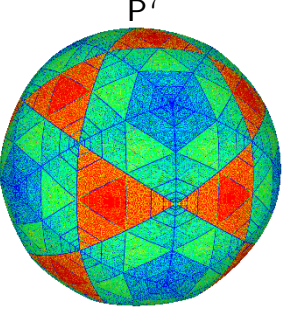

$\mathrm{H}$

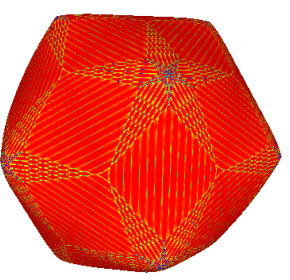

$\mathrm{P}^{4} \mathrm{H}^{4}$

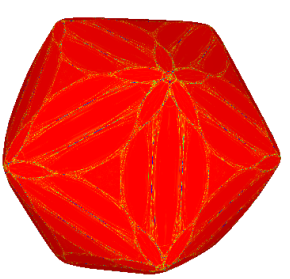

$\mathrm{P}^{2} \mathrm{H}^{2} \mathrm{P}^{2} \mathrm{H}^{2}$

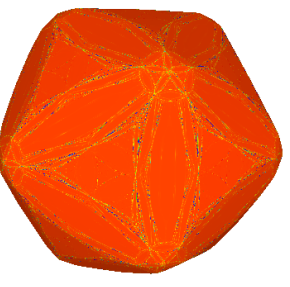

PHPHPHPH

Figure 17: Polyhedra generated by applying different compositions of 7 or 8 honeycomb and pushing operators from an icosahedron with pushing and honeycomb offsetting percentages being always 50 . They are all shown with reflection lines and discrete mean curvatures generated by MeshLab. 
[3] Boehm W., Müller A.: On de Casteljau's algorithm. Computer Aided Geometric Design, Volume 16, 587-605 (1999).

[4] de Boor C.: Cutting corners always works. Computer Aided Geometric Design, Volume 4(1-2), 125-131 (1987).

[5] de Boor C.: Local corner cutting and the smoothness of the limiting curve. Computer Aided Geometric Design, Volume 7(5): 389-397 (1990).

[6] de Boor C.: Convexity-preserving $C^{(1)}$ interpolation by corner cutting and stellation. Detailed, cleaned-up notes for a talk with the same title at the conference Math. Meth. for Curves and Surfaces, Biri June 1991. Unpublished manuscript.

[7] Chaikin G. M.: An algorithm for high-speed curve generation. Computer Graphics and Image Processing 3, 1974 pp 346-349.

[8] Claes J., Beets K., Van Reeth F.: A Corner-Cutting Scheme for Hexagonal Subdivision Surfaces. Geoff Wyvill (ed.) Proceedings Shape Modeling International 2002 pp.13-20.

[9] Dyn N., Levin D., Liu D.: Interpolatory convexity-preserving subdivision schemes for curves and surfaces. Computer-Aided Design, Volume 24, Issue 4, April 1992, pages 211-216.

[10] Gregory J. A., Qu R.: Nonuniform corner cutting Computer Aided Geometric Design, Volume 13: 763-772 (1996).

[11] Gardener R. J., Kallay M.: Subdivision and the Kernel of a Polyhedron. Discrete Comput. Geom., Volume 8: 417-427 (1992).

[12] Haase J. C. F.: Zur Theorie der ebenen Curven $n$-ter Ordnung mit $n-$ 1) $(n-2) / 2)$ Doppel- und Rückkehrpunkten. Math. Ann., Volume 2, 515-548 $(1870)$.

[13] Hu C.: Kantenschnittverfahren zur Erstellung glatter Flächen. Bachelor's Thesis. Karlsruher Institute for Technology (2018).

[14] Kobbelt L.: Sqrt 3-subdivision. In Proceedings of the 27th Annual Conference on Computer Graphics and Interactive Techniques, SIGGRAPH 2000, 103-112.

[15] Meyer M., Desbrun M., Schröder P., Barr A. H.: Discrete DifferentialGeometry Operators for Triangulated 2-Manifolds. In: Hege HC., Polthier K. (eds) Visualization and Mathematics III. Mathematics and Visualization. Springer, Berlin, Heidelberg (2003) 35-57.

[16] Paluszny M., Prautzsch H., Schäfer M.: A geometric look at corner cutting. Computer Aided Geometric Design, Volume 14, 1997, pages 421-447. 
[17] Prautzsch H.: Cutting edge refinement. Presentation at the 9th International conference on Curves and Surfaces, June 30, 2018 in Arcachon. (A paper with Yijun $\mathrm{Xu}$ is in preparation.)

[18] Prautzsch H., Boehm W.: Boxsplines. In: The Handbook of Computer Aided Geometric Design, Farin, Hoschek, Kim (eds.), North-Holland, 2002.

[19] Rham G. de.: Un peu de mathematique á propos d'une courbe plane.Elem. Math, Volume 2: 73-76 (1947).

[20] Song Z.: Interpolatory Convexity Preserving Subdivision Algorithms for Polyhedra. Master's Thesis. Karlsruher Institute for Technology (2018).

[21] Tóth F.: Sequences of polyhedra.Amer. Math. Monthly, 88: 145-146 (1981).

[22] Zhang H., Wang G.: Honeycomb Subdivision. Journal of Software, Volume 13: 1-9 (2002). 